Article

\title{
Biological Activities and Phytochemical Profiles of Extracts from Different Parts of Bamboo (Phyllostachys pubescens)
}

\author{
Akinobu Tanaka ${ }^{1, \dagger}$, Qinchang Zhu ${ }^{1, \dagger}$, Hui Tan ${ }^{1}$, Hiroki Horiba ${ }^{1}$, Koichiro Ohnuki ${ }^{2}$, \\ Yasuhiro Mori ${ }^{3}$, Ryoko Yamauchi ${ }^{4}$, Hiroya Ishikawa ${ }^{4}$, Akira Iwamoto ${ }^{5}$, Hiroharu Kawahara ${ }^{5}$ \\ and Kuniyoshi Shimizu ${ }^{1, *}$
}

1 Department of Agro-environmental Sciences, Faculty of Agriculture, Kyushu University, 6-10-1 Hakozaki, Higashi-ku, Fukuoka, 812-8581, Japan;

E-Mails: akinobu0917@gmail.com (A.T.); zhu_qinchang@hotmail.com (Q.Z.); thth229@agr.kyushu-u.ac.jp (H.T.); h2.horiba0728@agr.kyushu-u.ac.jp (H.H.)

Department of Biological and Environmental Chemistry, Kinki University, Kayanomori 11-6, Iizuka, Fukuoka, 820-8555, Japan; E-Mail: ohnuki_usi@yahoo.co.jp

3 Fukuoka Prefecture Institute of Agricultural and Forest Resources, 1438-2, Toyota, Yamamoto-town, Kurume, Fukuoka, 839-0827, Japan; E-Mail: mori-y9360@ffrec.pref.fukuoka.jp

4 International College of Arts and Sciences, Fukuoka Women's University, Fukuoka 813-8529, Japan; E-Mails: yamauchi-r@fwu.ac.jp (R.Y.); ishikawa@fwu.ac.jp (H.I.)

5 Department of Materials Science and Chemical Engineering, Kitakyushu National College of Technology, 5-20-1 Shii, Kokuraminami-ku, Kitakyushu, Fukuoka, 802-0985, Japan;

E-Mails: iwamoto.akira.344@s.kyushu-u.ac.jp (A.I.); hk128@kct.ac.jp (H.K.)

$\dagger$ Authors contributed equally to this work.

* Author to whom correspondence should be addressed; E-Mail: shimizu@agr.kyushu-u.ac.jp; Tel./Fax: +81-92-642-3002.

Received: 8 April 2014; in revised form: 16 May 2014 / Accepted: 23 May 2014 / Published: 18 June 2014

\begin{abstract}
Besides being a useful building material, bamboo also is a potential source of bioactive substances. Although some studies have been performed to examine its use in terms of the biological activity, only certain parts of bamboo, especially the leaves or shoots, have been studied. Comprehensive and comparative studies among different parts of bamboo would contribute to a better understanding and application of this knowledge. In this study, the biological activities of ethanol and water extracts from the leaves, branches, outer culm, inner culm, knots, rhizomes and roots of Phyllostachys pubescens, the major
\end{abstract}


species of bamboo in Japan, were comparatively evaluated. The phytochemical profiles of these extracts were tentatively determined by liquid chromatography-mass spectrometry (LC-MS) analysis. The results showed that extracts from different parts of bamboo had different chemical compositions and different antioxidative, antibacterial and antiallergic activities, as well as on on melanin biosynthesis. Outer culm and inner culm were found to be the most important sources of active compounds. 8-C-Glucosylapigenin, luteolin derivatives and chlorogenic acid were the most probable compounds responsible for the anti-allergy activity of these bamboo extracts. Our study suggests the potential use of bamboo as a functional ingredient in cosmetics or other health-related products.

Keywords: Phyllostachys pubescens; cosmetics; anti-melanogenesis; antioxidation; antibacterial; anti-allergy; HPLC; LC-MS-IT-TOF

\section{Introduction}

Bamboo is well known for its extensive use. Besides being used in building construction, its roots and leaves have been used medicinally. Studies have revealed that bamboo leaves have antioxidant, anticancer and antibiotic properties [1,2]. In previous studies, various active compounds, such as flavones, glycosides, phenolic acids, coumarin lactones, anthraquinones and amino acids, have been isolated from the leaves [3-7]. 2, 6-Dimethoxy- $p$-benzoquinone isolated from the skin of bamboo trees and two chitin-binding peptides (Pp-AMP1 and Pp AMP2) isolated from bamboo shoots were found to have antibiotic activities [8,9]. Stigmasterol and dihydrobrassicasterol isolated from the skin of bamboo shoot showed antibacterial activity [10], as well as tricin and taxifolin [11].

Phyllostachys pubescens (P. pubescens) is the major species of bamboo in Japan, which is widely distributed through the country. In fact, how to stop its further spread is a problem in Japan [12]. Hence, new applications of bamboo in various commercial industries are being explored. Several studies have been performed to demonstrate the use of P. pubescens in terms of its biological activity. These studies have mainly focused on extracts from specific parts of $P$. pubescens. For example, the antioxidant activity of leaves [13] and shoots [14], antiallegic [15] and anticancer [16] activities of leaves and branches and antibacterial activities of stems [8], shoots [9], and shoot skins [17]. However, comprehensive and comparative studies of extracts from all parts of bamboo using the same extraction solvent have not been done. In this study, $P$. pubescens was separated into 10 parts, including leaves, branches, outer culm ( $5 \mathrm{~m}$ and $1 \mathrm{~m}$ above the ground, respectively), inner culm ( $5 \mathrm{~m}$ and $1 \mathrm{~m}$ above the ground, respectively), knots ( $5 \mathrm{~m}$ and $1 \mathrm{~m}$ above the ground, respectively), rhizomes and roots (Figure 1). These parts of bamboo were extracted by ethanol and hot water. All resulting extracts were subjected to four assays for bioactivities that are usually of interest to the cosmetics industry. They are melanin synthesis assay, antioxidant assay, antibacterial assay and antiallergic assay. At the same time, the chromatographic profiles of these extracts were determined and their components were partially identified using liquid chromatography-mass spectrometry (LC-MS). Through these tests and analysis, the potential use of bamboo in health-related industries, especially in cosmetics industry was evaluated. 


\section{Results and Discussion}

In the present study, the ethanol and hot water extracts of various parts of $P$. pubescens (Figure 1) were examined for several biological activities suing the melanin biosynthesis assay (Table 1), antioxidant assay (Table 2), antibacterial assay (Table 3) and immunoglobulin E (IgE) production assay (Table 4). Their phytochemical profiles were also investigated through LC-MS analysis (Tables 5, 6 and Figures S1-S3).

Figure 1. Parts of $P$. pubescens used in present study. $P$. pubescens plants were separated into leaves, branches, outer culm, inner culm, knots, rhizomes and roots. Outer culm, inner culm and knot samples were obtained separately at the height of $5.0 \pm 0.3$ and $1.0 \pm 0.3$ meter above ground level.

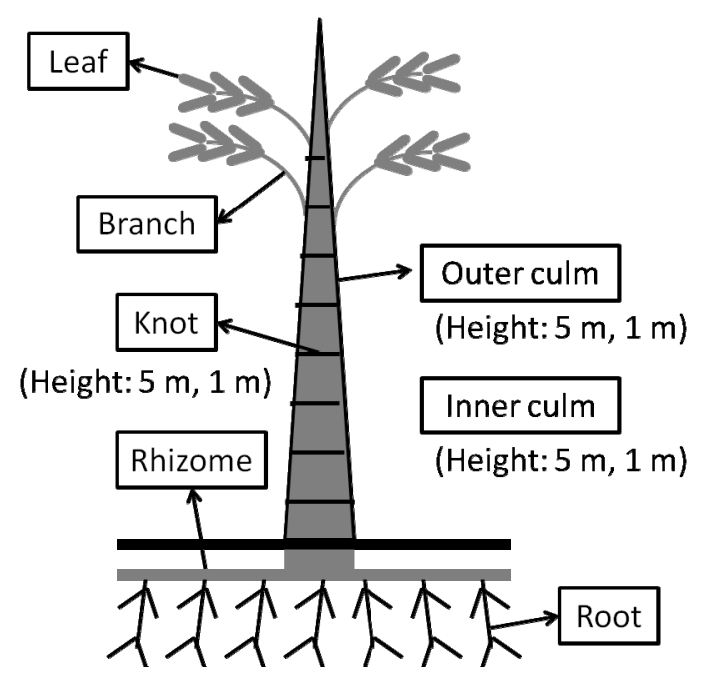

\subsection{Activity on Melanin Biosynthesis}

Table 1 shows the effect of the ethanol extracts and the hot water extracts of $P$. pubescens on melanin biosynthesis and cell proliferation of B16 melanoma cells. After treating with different concentrations of the extract for 3 days, B16 melanoma cells were examined for cell viability (CV) and melanin content (MC). The cell viability was measured by the classic MTT assay, while the melanin content was determined by the absorbance at $405 \mathrm{~nm}$. One important concept when selecting bioactive extracts that modulate skin pigmentation for cosmetics is that they should have minimal effects on cell proliferation and/or survival.

As shown in Table 1, the ethanol extracts of branches, and outer culm (5 m, $1 \mathrm{~m})$ showed melanin biosynthesis inhibitory activity (Type A) in a dose-dependent manner. The ethanol extract of branches inhibited biosynthesis of melanin at $120 \mu \mathrm{g} / \mathrm{mL}$ (CV was $86.3 \%$ and $\mathrm{MC}$ was $56.0 \%$ ). The ethanol extract of the outer culm at $5 \mathrm{~m}$ showed activity at 120 and $60 \mu \mathrm{g} / \mathrm{mL}$ (CVs were $86.9 \%$ and $98.9 \%$; MCs were 44.4 and $72.2 \%$, respectively). The ethanol extract of the outer culm at $1 \mathrm{~m}$ showed activity at 120 and $60 \mu \mathrm{g} / \mathrm{mL}$ (CVs were 124 and 109\%; MCs were 49.5 and 79.8\%, respectively). On the other hand, the ethanol extracts of the inner culm at both heights $(5 \mathrm{~m}, 1 \mathrm{~m})$, knots at $1 \mathrm{~m}$, rhizomes and roots showed selective melanin biosynthesis-stimulating activity (Type B). The ethanol extract of the inner culm at $5 \mathrm{~m}$ stimulated biosynthesis of melanin at $60 \mu \mathrm{g} / \mathrm{mL}(\mathrm{CV}$ was $88.5 \%$ and $\mathrm{MC}$ was 
$109 \%$ ). Also, the ethanol extract of the inner culm at $1 \mathrm{~m}$ stimulated biosynthesis of melanin at 120, 60 and $20 \mu \mathrm{g} / \mathrm{mL}$ (CVs were 106\%, 106\% and 103\%; MCs were 142\%, 151\% and 134\%, respectively). The ethanol extract of knots at $1 \mathrm{~m}$ showed activity at 120 and $60 \mu \mathrm{g} / \mathrm{mL}(\mathrm{CVs}$ were $100 \%$ and $97.0 \%$; MCs were $133 \%$ and $119 \%$, respectively).

Table 1. Effect of the (a) ethanol extracts and (b) the hot water extracts of P. pubescens on melanin biosynthesis and cell proliferation of B16 melanoma cells.

(a)

\begin{tabular}{|c|c|c|c|c|c|c|c|c|c|}
\hline \multirow{3}{*}{ Part } & \multicolumn{9}{|c|}{ Ethanol extract } \\
\hline & \multicolumn{3}{|l|}{$120 \mu \mathrm{g} / \mathrm{mL}$} & \multicolumn{3}{|l|}{$60 \mu \mathrm{g} / \mathrm{mL}$} & \multicolumn{3}{|l|}{$20 \mu \mathrm{g} / \mathrm{mL}$} \\
\hline & $\mathrm{CV}$ & MC & Type & CV & MC & Type & $\mathrm{CV}$ & MC & Type \\
\hline Leaf & $91.2 \pm 1.06$ & $105 \pm 5.52$ & - & $96.7 \pm 8.10$ & $99.0 \pm 8.53$ & - & $101 \pm 1.98$ & $103 \pm 1.32$ & - \\
\hline Branch & $86.3 \pm 1.71$ & $56.0 \pm 7.90$ & $\mathrm{~A}, \mathrm{C}$ & $91.0 \pm 2.74$ & $75.6 \pm 3.52$ & - & $90.2 \pm 0.84$ & $84.6 \pm 2.09$ & - \\
\hline Outer culm (5 m) & $86.9 \pm 10.1$ & $44.4 \pm 5.64$ & $\mathrm{~A}, \mathrm{C}$ & $98.9 \pm 0.98$ & $72.2 \pm 1.55$ & A & $112.9 \pm 4.27$ & $104 \pm 2.34$ & - \\
\hline Outer culm (1 m) & $124 \pm 9.08$ & $49.5 \pm 5.38$ & A & $109 \pm 1.20$ & $79.8 \pm 3.19$ & A & $124 \pm 9.08$ & $112 \pm 2.97$ & - \\
\hline Inner culm (5 m) & $98.8 \pm 2.09$ & $110 \pm 2.67$ & - & $88.5 \pm 10.5$ & $109 \pm 9.68$ & $\mathrm{~B}, \mathrm{C}$ & $98.0 \pm 1.09$ & $106 \pm 8.01$ & - \\
\hline Inner culm (1 m) & $106 \pm 1.80$ & $142 \pm 2.87$ & $\mathrm{~B}$ & $106 \pm 5.63$ & $151.9 \pm 9.59$ & B & $103 \pm 3.32$ & $134 \pm 3.59$ & B \\
\hline $\operatorname{Knot}(5 \mathrm{~m})$ & $93.6 \pm 5.51$ & $101 \pm 9.90$ & - & $90.5 \pm 1.96$ & $104 \pm 0.69$ & - & $93.4 \pm 4.86$ & $101 \pm 3.27$ & - \\
\hline Knot (1 m) & $100 \pm 6.22$ & $133 \pm 20.0$ & B & $97.0 \pm 7.14$ & $119 \pm 2.97$ & $\mathrm{~B}$ & $96.8 \pm 6.46$ & $107 \pm 6.67$ & - \\
\hline Rhizome & $120 \pm 2.97$ & $137 \pm 19.0$ & - & $118 \pm 1.88$ & $144 \pm 21.6$ & B & $114 \pm 4.44$ & $121 \pm 4.2$ & - \\
\hline Root & $91.5 \pm 2.68$ & $118 \pm 6.78$ & B & $88.6 \pm 1.2$ & $126 \pm 5.53$ & $\mathrm{~B}, \mathrm{C}$ & $104 \pm 10.1$ & $111 \pm 12.7$ & - \\
\hline
\end{tabular}

(b)

\begin{tabular}{|c|c|c|c|c|c|c|c|c|c|}
\hline \multirow{3}{*}{ Part } & \multicolumn{9}{|c|}{ Hot water extract } \\
\hline & \multicolumn{3}{|l|}{$120 \mu \mathrm{g} / \mathrm{mL}$} & \multicolumn{3}{|l|}{$60 \mu \mathrm{g} / \mathrm{mL}$} & \multicolumn{3}{|l|}{$20 \mu \mathrm{g} / \mathrm{mL}$} \\
\hline & $\mathrm{CV}$ & MC & Type & CV & MC & Type & CV & MC & Type \\
\hline Leaf & $109 \pm 9.06$ & $114 \pm 7.71$ & - & $107 \pm 8.35$ & $108 \pm 11.1$ & - & $109 \pm 9.06$ & $143.8 \pm 2.22$ & $\mathrm{~B}$ \\
\hline Branch & $79.7 \pm 10.7$ & $84.8 \pm 8.16$ & $\mathrm{C}$ & $77.3 \pm 3.67$ & $87.2 \pm 1.39$ & $\mathrm{C}$ & $83.1 \pm 7.42$ & $109 \pm 2.73$ & $\mathrm{~B}, \mathrm{C}$ \\
\hline Outer culm (5 m) & $121 \pm 6.83$ & $78.9 \pm 6.10$ & $\mathrm{~A}$ & $125 \pm 3.76$ & $93.3 \pm 13.0$ & A & $138 \pm 2.22$ & $94.6 \pm 6.76$ & A \\
\hline Outer culm $(1 \mathrm{~m})$ & $118 \pm 2.81$ & $125 \pm 16.5$ & - & $114 \pm 2.99$ & $104 \pm 16.5$ & - & $116 \pm 6.16$ & $117 \pm 14.8$ & - \\
\hline Inner culm (5 m) & $97.5 \pm 7.10$ & $104 \pm 6.62$ & - & $114 \pm 9.85$ & $105 \pm 15.2$ & - & $96.2 \pm 9.62$ & $101 \pm 2.82$ & - \\
\hline Inner culm (1 m) & $120 \pm 5.10$ & $113 \pm 17.6$ & - & $110 \pm 15.0$ & $108 \pm 0.28$ & - & $113 \pm 0.76$ & $102 \pm 1.97$ & - \\
\hline $\operatorname{Knot}(5 \mathrm{~m})$ & $79.1 \pm 6.23$ & $94.8 \pm 5.69$ & $\mathrm{C}$ & $85.8 \pm 2.81$ & $109 \pm 9.61$ & $\mathrm{~B}, \mathrm{C}$ & $77.7 \pm 1.65$ & $101 \pm 1.86$ & $\mathrm{~B}, \mathrm{C}$ \\
\hline Knot (1 m) & $90.1 \pm 12.9$ & $99.5 \pm 14.1$ & - & $103 \pm 17.5$ & $103 \pm 22.0$ & - & $78.0 \pm 8.30$ & $96.8 \pm 2.91$ & $\mathrm{C}$ \\
\hline Rhizome & $111 \pm 3.12$ & $88.9 \pm 2.01$ & A & $123 \pm 9.64$ & $97.3 \pm 15.3$ & A & $115 \pm 7.03$ & $93.2 \pm 12.6$ & A \\
\hline Root & $99.9 \pm 2.22$ & $114 \pm 10.4$ & - & $94.3 \pm 4.94$ & $121 \pm 9.18$ & $\mathrm{~B}$ & $98.2 \pm 3.49$ & $116 \pm 11.2$ & $\mathrm{~B}$ \\
\hline
\end{tabular}

Data presented as means $\pm \mathrm{SD}(\mathrm{n}=3)$. $\mathrm{CV}$, cell viability $(\%)$; MC, melanin content $(\%)$. Type A $(\mathrm{CV}-\mathrm{MC} \geq 20)$ : melanin-biosynthesis-inhibitory activity; Type B (MC-CV $\geq 20)$ : melanin-biosynthesis-stimulating activity; Type $\mathrm{C}(\mathrm{CV} \leq 90 \%)$ : cytotoxicity. Arbutin $(100 \mu \mathrm{g} / \mathrm{mL})$ was used as the positive control for melanin-biosynthesis inhibition. Its $\mathrm{CV}$ and $\mathrm{MC}$ were $94.7 \%$ and $46.5 \%$, respectively. It belongs to the Type A.

It is notable that ethanol extracts of knots from $1 \mathrm{~m}$ but not from $5 \mathrm{~m}$ showed activity. Also, ethanol extracts of rhizomes $(60 \mu \mathrm{g} / \mathrm{mL})$ and roots $(120$ and $60 \mu \mathrm{g} / \mathrm{mL})$ showed melanin-biosynthesisstimulating activity (CV was $118 \%$ and $\mathrm{MC}$ was $144 \%$ for rhizomes; $\mathrm{CV}$ s were $91.5 \%$ and $88.6 \%$ and MCs were $118 \%$ and $126 \%$ for roots, respectively). In this assay, DMSO was used to dissolve ethanol extracts, and its final concentration was $0.2 \%$. Under such concentration, DMSO didn't show 
cytotoxicity to the B16 melanoma cells (MTT assay, data not shown). Because the results were calculated basing on the comparison with DMSO-treated group, DMSO used in this assay should not affect the results.

Table 2. Antioxidant activity of the ethanol extracts and hot water extracts from P. pubescens.

\begin{tabular}{|c|c|c|c|c|c|c|}
\hline \multirow[b]{2}{*}{ Part } & \multicolumn{3}{|c|}{ Ethanol Extract } & \multicolumn{3}{|c|}{ Hot Water Extract } \\
\hline & $\begin{array}{l}\text { ORAC } \\
(\mathrm{mgTE} / \mathrm{mg})\end{array}$ & 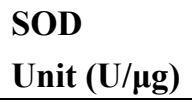 & $\begin{array}{l}\text { ABTS } \\
\mathrm{IC}_{50}(\mu \mathrm{g} / \mathrm{mL})\end{array}$ & $\begin{array}{l}\text { ORAC } \\
(\mathrm{mgTE} / \mathrm{mg})\end{array}$ & 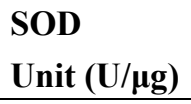 & $\begin{array}{l}\text { ABTS } \\
\operatorname{IC}_{50}(\mu \mathrm{g} / \mathrm{mL})\end{array}$ \\
\hline Leaf & $0.07 \pm 0.02$ & nd & nd & $0.37 \pm 0.08$ & nd & $306.7 \pm 5.7$ \\
\hline Branch & $0.69 \pm 0.04$ & $4.4 \pm 1.0$ & $350.6 \pm 7.1$ & $0.84 \pm 0.01$ & $0.6 \pm 0.0$ & $179.5 \pm 3.6$ \\
\hline Outer culm (5 m) & $0.52 \pm 0.07$ & $0.2 \pm 0.0$ & nd & $0.65 \pm 0.03$ & $1.0 \pm 0.3$ & $113.7 \pm 18.2$ \\
\hline Outer culm (1 m) & $0.18 \pm 0.01$ & $0.1 \pm 0.0$ & nd & $0.59 \pm 0.05$ & $0.8 \pm 0.1$ & $140.1 \pm 1.4$ \\
\hline Inner culm (5 m) & $0.72 \pm 0.09$ & $0.9 \pm 0.1$ & $88.5 \pm 0.8$ & $0.29 \pm 0.03$ & nd & $198.3 \pm 3.0$ \\
\hline Inner culm (1 m) & $1.35 \pm 0.14$ & $0.2 \pm 0.0$ & $373.8 \pm 3.2$ & $0.30 \pm 0.00$ & nd & $231.9 \pm 4.9$ \\
\hline Knot (5 m) & $0.22 \pm 0.00$ & nd & nd & $0.29 \pm 0.02$ & nd & $245.0 \pm 4.2$ \\
\hline Knot (1 m) & $0.22 \pm 0.00$ & nd & nd & $0.28 \pm 0.01$ & nd & $240.7 \pm 1.9$ \\
\hline Rhizome & $0.71 \pm 0.02$ & $0.1 \pm 0.0$ & $171.5 \pm 5.4$ & $0.31 \pm 0.00$ & nd & $266.7 \pm 6.8$ \\
\hline Root & $0.05 \pm 0.03$ & nd & nd & $0.54 \pm 0.02$ & $0.2 \pm 0.0$ & $209.7 \pm 7.8$ \\
\hline
\end{tabular}

Data presented as means \pm SD $(\mathrm{n}=3)$; ORAC, oxygen radical absorbance capacity; SOD, superoxide dismutase; ABTS, 2,2'-azino-bis (3-ethylbenzothiazoline-6-sulphonic acid); ORAC values are expressed as relative Trolox equivalents per milligram; nd, not determined because the value is below the detection limit.

Table 3. Antibacterial activity of the ethanol extracts and the hot water extracts of P. pubescens.

\begin{tabular}{|c|c|c|c|c|c|c|}
\hline \multirow{3}{*}{ Part } & \multicolumn{3}{|c|}{ Ethanol Extract } & \multicolumn{3}{|c|}{ Hot Water Extract } \\
\hline & \multicolumn{2}{|c|}{ Growth Inhibition } & \multirow{2}{*}{$\begin{array}{c}\mathrm{MIC} / \mathrm{MBC} \\
(\mu \mathrm{g} / \mathrm{mL})\end{array}$} & \multicolumn{2}{|c|}{ Growth Inhibition } & \multirow{2}{*}{$\begin{array}{c}\mathrm{MIC} / \mathrm{MBC} \\
(\mu \mathrm{g} / \mathrm{mL})\end{array}$} \\
\hline & $\begin{array}{c}\text { Concentration } \\
(\mu \mathrm{g} / \mathrm{mL})\end{array}$ & $\begin{array}{c}\text { Rate }(\% v s . \\
\text { Control) * }\end{array}$ & & $\begin{array}{c}\text { Concentration } \\
(\mu \mathrm{g} / \mathrm{mL})\end{array}$ & $\begin{array}{c}\text { Rate }(\% \text { vs. } \\
\text { Control) } *\end{array}$ & \\
\hline Leaf & 600 & - & - & 600 & $98.1 \pm 0.47$ & $1200 / 1600$ \\
\hline Branch & 1200 & - & - & 1200 & $97.6 \pm 1.61$ & $1400 />1400$ \\
\hline Outer culm $(5 \mathrm{~m})$ & 600 & $97.8 \pm 11.6$ & $400 / 1600$ & 600 & $13.7 \pm 6.89$ & nd \\
\hline Outer culm $(1 \mathrm{~m})$ & 600 & $100 \pm 0.47$ & $400 / 1600$ & 600 & $12.1 \pm 9.30$ & nd \\
\hline Inner culm (5 m) & 600 & - & - & 600 & $99.5 \pm 1.68$ & $>1600$ \\
\hline Inner culm (1 m) & 600 & - & - & 600 & - & - \\
\hline $\operatorname{Knot}(5 \mathrm{~m})$ & 600 & - & - & 600 & $31.2 \pm 15.0$ & nd \\
\hline $\operatorname{Knot}(1 \mathrm{~m})$ & 600 & - & - & 600 & - & - \\
\hline Rhizome & 1200 & - & - & 1200 & $44.1 \pm 12.9$ & nd \\
\hline Root & 1200 & - & - & 1200 & $52.4 \pm 15.7$ & nd \\
\hline
\end{tabular}

* Data presented as means $\pm \mathrm{SD}(\mathrm{n}=3)$. MIC: minimum inhibitory concentration; MBC: minimum bactericidal concentration. -: no antibacterial activity; nd: non-detect. Sorbic acid $(200 \mu \mathrm{g} / \mathrm{mL})$ was used as positive control and its inhibition rate was $73.7 \% \pm 10.7 \%$. 
Table 4. Anti-allergy activity of the ethanol extracts and the hot water extracts of P. pubescens.

\begin{tabular}{lll}
\hline \multirow{2}{*}{ Part } & \multicolumn{2}{l}{ IgE Production (\%) } \\
\cline { 2 - 3 } & Ethanol Extract & Hot Water Extract \\
\hline Leaf & $97.3 \pm 38.9$ & $57.2 \pm 9.28^{* *}$ \\
Branch & $227 \pm 95.8$ & $103 \pm 45.4$ \\
Outer culm $(5 \mathrm{~m})$ & $144 \pm 27.7$ & $70.7 \pm 13.1^{*}$ \\
Outer culm $(1 \mathrm{~m})$ & $137 \pm 109$ & $64.1 \pm 6.47^{* *}$ \\
Inner culm $(5 \mathrm{~m})$ & $110 \pm 39.6$ & $64.1 \pm 18.1^{*}$ \\
Inner culm $(1 \mathrm{~m})$ & $93.1 \pm 15.0$ & $66.9 \pm 19.8$ \\
Knot $(5 \mathrm{~m})$ & $115 \pm 61.9$ & $64.1 \pm 10.3^{* *}$ \\
Knot $(1 \mathrm{~m})$ & $107 \pm 39.8$ & $73.8 \pm 14.8$ \\
Rhizome & $60.6 \pm 29.8$ & $62.8 \pm 15.4 *$ \\
Root & $75.1 \pm 31.0$ & $73.7 \pm 19.4$ \\
\hline
\end{tabular}

Date presented as means $\pm \mathrm{SD}(\mathrm{n}=3)$. Concentration of each sample is $60 \mu \mathrm{g} / \mathrm{mL}$. Significant differences between control and each extract were determined by Student's t-test: $* p<0.05,{ }^{* *} p<0.01$.

The hot water extracts of the outer culm $(5 \mathrm{~m})$ and rhizomes showed melanin-biosynthesisinhibitory activity (Type A behavior) at 120, 60 and $20 \mu \mathrm{g} / \mathrm{mL}$ (CVs were 121\%, 125\% and 138\% and MCs were 78.9, 93.3 and 94.6\%, respectively for outer culm at $5 \mathrm{~m}$; CVs were $111 \%, 123 \%$ and $115 \%$, and MCs were $88.9 \%, 97.3 \%$ and $93.2 \%$, for rhizomes). On the other hand, the hot water extracts of leaves, branches, knots at $5 \mathrm{~m}$, and roots showed melanin-biosynthesis-stimulating activity (Type B behavior). The hot water extract of leaves showed activity at $20 \mu \mathrm{g} / \mathrm{mL}$ (CV was $109 \%$ and MC was $143 \%$ ). The hot water extract of branches showed activity at $20 \mu \mathrm{g} / \mathrm{mL}$ (CV was $83.1 \%$ and MC was $109 \%$ ). The latter extract showed relatively strong cytotoxicity at tested concentrations and was classified as type $\mathrm{C}$. The hot water extract of knots at $5 \mathrm{~m}$ showed activity at 60 and $20 \mu \mathrm{g} / \mathrm{mL}$ (CVs were 85.8\% and 77.7\%; MCs were 109\% and 101\%, respectively). This extract also showed relatively strong cytotoxicity at tested concentrations and was classified as type C (CVs were $79.1 \%$, $85.8 \%$ and $77.7 \%$, respectively). The hot water extract of roots showed activity at 60 and $20 \mu \mathrm{g} / \mathrm{mL}$ (CVs were $94.3 \%$ and $98.2 \%$; MCs were $121 \%$ and $116 \%$, respectively).

The melanin-biosynthesis-inhibition activity of extract prepared from bamboo indicates its potential use as a skin-whitening agent. On the other hand, melanin-biosynthesis-stimulating activity is important for skin tanning agent and hair dyes.

\subsection{Antioxidant Activity}

Table 2 shows the antioxidant activity of the ethanol extracts and the hot water extracts of $P$. pubescens. The ethanol extract of the inner culm at $1 \mathrm{~m}$ showed the highest ORAC value (1.35 $\mathrm{mgTE} / \mathrm{mg})$ in all tested extracts. Other extracts showed ORAC values from 0.07 to 0.84 $\mathrm{mgTE} / \mathrm{mg}$. SOD-like activities were detected from several extracts. The ethanol extract of branches showed the strongest SOD-like activity $(4.4 \mathrm{U} / \mu \mathrm{g})$. Also, the ethanol extracts of the outer culm at both heights, inner culm at both heights, and rhizomes and the hot water extracts of branches, outer culm at both heights and roots showed SOD-like activities $(0.1-1.0 \mathrm{U} / \mu \mathrm{g})$. The ethanol extract of the inner culm at $5 \mathrm{~m}$ showed the strongest ABTS radical decolorization activity in all tested extracts $\left(\mathrm{IC}_{50}=88.5 \mu \mathrm{g} / \mathrm{mL}\right)$. The 
$\mathrm{IC}_{50} \mathrm{~s}$ could be calculated from all hot water extracts. However, among the ethanol extracts, only those of the branches, inner culm at both heights, and rhizomes showed enough activity to calculate their $\mathrm{IC}_{50} \mathrm{~s}$. The hot water extracts tended to show stronger activity than the ethanol extracts. Skin is a major potential target of oxidative stress. Oxidative stress enhances melanin biosynthesis, damages DNA, and may induce proliferation of melanocytes [18]. Therefore, antioxidants can reduce hyperpigmentation. Considering both the melanin-biosynthesis-inhibiting and antioxidant activities of bamboo extracts, they have potential as skin-whitening agents.

There was no correlation between the intensity of ORAC, SOD and ABTS. This is not a surprising result, because these three assays evaluate the activity throughout quite different mechanisms. The ORAC assay is based on hydrogen atom transfer reactions and the ABTS inhibition rates are based on the electron-transfer ability of the sample's components. Also, SOD-like activity is based on the antioxidative enzyme-like activity of the sample's components.

\subsection{Antibacterial Activity}

Antibacterial activity against Staphylococcus aureus is an important attribute of skin cosmetics, because the proliferation of bacteria causes skin problems such as acne, comedo, papules, cellulitis and allergies [19,20]. Therefore, we also evaluated the antibacterial activity of the extracts from $P$. pubescens. Table 3 shows the antibacterial activity of the ethanol extracts and the hot water extracts of P. pubescens. The ethanol extracts of the outer culm at both heights and the hot water extracts of leaves, branches and inner culm at $5 \mathrm{~m}$ almost completely inhibited the growth of bacteria (growth inhibition rates were 97.8, 100, 98.1, 97.6 and 99.5, respectively). For the part of outer culm at the height of both $5 \mathrm{~m}$ and $1 \mathrm{~m}$, the ethanol extracts showed strong antibacterial activity (growth inhibition rates were $97.8 \%$ and $100 \%$ for 5 and $1 \mathrm{~m}$, respectively), while the hot water extract didn't show good activity (growth inhibition rates were $13.7 \%$ and $12.1 \%$ ), suggesting that the antibacterial constituents in the outer culm are lipophilic The MIC (minimum inhibitory concentration) and MBC (minimum bactericidal concentration) of active extracts were further determined using higher concentrations. The ethanol extracts of outer culm at both heights of $5 \mathrm{~m}$ and $1 \mathrm{~m}$ showed the lowest MIC $(400 \mu \mathrm{g} / \mathrm{mL})$. Three extracts showed minimum bacterialcidal effect at $1,600 \mu \mathrm{g} / \mathrm{mL}$. They are ethanol extracts of outer culm $(5 \mathrm{~m}$ and $1 \mathrm{~m})$ and hot water extract of leaf. For the hot water extracts that showed weaker activity, the MIC/MBC were not detected because of the low activity of them at the concentrations close to their maximum solubility. Most of hot water extracts showed antibacterial activity at various inhibition rates $(12.1 \%-100 \%)$. However, among the ethanol extracts, only the outer culm at 5 and $1 \mathrm{~m}$ showed antibacterial activity. The hot water extracts tended to show stronger antibacterial activity than the ethanol extracts. The antibacterial activity of bamboo would be useful in keeping skin healthy.

\subsection{Anti-Allergy Activity}

Some components in cosmetics cause side effect of allergies, the addiction of ingredients with antiallergy activity to the cosmetics will be helpful to avoid such side effect. 
Table 5. Partial characterization of ethanol extracts of various parts of bamboo by LCMS-IT-TOF.

\begin{tabular}{|c|c|c|c|c|c|c|c|}
\hline \multirow[b]{2}{*}{ Part } & \multirow[b]{2}{*}{ Comp. * } & \multirow{2}{*}{$\begin{array}{l}\mathrm{tR} \\
(\mathrm{min})\end{array}$} & \multirow{2}{*}{$\begin{array}{l}\mathrm{UV} \lambda \\
(\mathrm{nm})\end{array}$} & \multicolumn{2}{|l|}{ MS } & \multirow[b]{2}{*}{ MS/MS } & \multirow[b]{2}{*}{$\begin{array}{l}\text { Tentative } \\
\text { Identification }\end{array}$} \\
\hline & & & & {$[\mathbf{M}+\mathbf{H}]^{+}$} & $\begin{array}{l}\text { Main } \\
\text { Fragments }\end{array}$ & & \\
\hline \multirow{5}{*}{ Leaf } & 1 & 7.79 & 254,326 & 595.1222 & $\begin{array}{l}563.2069 \\
385.1727 \\
401.2092\end{array}$ & $\begin{array}{l}472.1109 \\
325.0804 \\
457.1063 \\
379.0754 \\
\end{array}$ & $\begin{array}{l}\text { Di- } C, C \text {-hexosyl } \\
\text { apigenin }\end{array}$ \\
\hline & 2 & 9.75 & 254,326 & 583.2920 & $\begin{array}{l}249.1044 \\
331.6180 \\
419.1789 \\
532.1011 \\
\end{array}$ & - & Tricin derivative \\
\hline & 3 & 10.99 & 254,326 & 547.0304 & $\begin{array}{l}214.9922 \\
405.2008 \\
316.5860 \\
474.0304 \\
\end{array}$ & $\begin{array}{l}391.0690 \\
260.0637 \\
419.1118\end{array}$ & Not identified \\
\hline & 4 & 13.68 & 254,326 & 639.1865 & $\begin{array}{l}561.3183 \\
427.5450 \\
357,1133 \\
331.0914 \\
\end{array}$ & 331.0833 & $\begin{array}{l}O \text {-hexosyl-O- } \\
\text { deoxyhexosyl } \\
\text { tricin }\end{array}$ \\
\hline & 5 & 13.96 & 254,326 & 493.1227 & $\begin{array}{l}235.0158 \\
314.0666 \\
\end{array}$ & 331.0777 & $O$-hexosyl tricin \\
\hline Branch & 6 & 11.01 & 254,326 & 433.1361 & $\begin{array}{l}313.0428 \\
214.9618\end{array}$ & $\begin{array}{l}283.0601 \\
337.0809 \\
415.0739 \\
162.9025 \\
\end{array}$ & $\begin{array}{l}\text { 6- } C \text {-glucosyl } \\
\text { apigenin } \\
\text { (isovitexin) }\end{array}$ \\
\hline \multirow{3}{*}{$\begin{array}{l}\text { Outer culm } \\
(5 \mathrm{~m})\end{array}$} & 7 & 4.48 & 254 & 351.0937 & $\begin{array}{l}196.9942 \\
442.0793 \\
253.0631 \\
156.0012 \\
\end{array}$ & $\begin{array}{l}269.3353 \\
315.1813 \\
211.5120 \\
153.9859 \\
\end{array}$ & Not identified \\
\hline & 1 & 7.55 & 254,326 & 595.2048 & $\begin{array}{l}401.1621 \\
563.2534 \\
385.1795 \\
511.1788 \\
\end{array}$ & $\begin{array}{l}383.1592 \\
373.1058 \\
318.5544 \\
244.3389 \\
\end{array}$ & $\begin{array}{l}\text { Di- } C, C \text {-hexosyl } \\
\text { apigenin }\end{array}$ \\
\hline & 4 & 13.65 & 254,326 & 639.1805 & $\begin{array}{l}561.3528, \\
589.1020 \\
315.0292 \\
173.9611 \\
\end{array}$ & $\begin{array}{l}331.0775 \\
270.0903 \\
415.4247\end{array}$ & $\begin{array}{l}O \text {-hexosyl- } O \text { - } \\
\text { deoxyhexosyl } \\
\text { tricin }\end{array}$ \\
\hline \multirow{2}{*}{$\begin{array}{l}\text { Outer culm } \\
(1 \mathrm{~m})\end{array}$} & 1 & 7.70 & 254,326 & 595.1777 & $\begin{array}{l}563.2181 \\
385.2050 \\
457.1007 \\
214.9845\end{array}$ & $\begin{array}{l}325.0885 \\
427.1041 \\
457.0921 \\
379.0553\end{array}$ & $\begin{array}{l}\text { Di- } C, C \text {-hexosyl } \\
\text { apigenin }\end{array}$ \\
\hline & 8 & 9.76 & 254,326 & 582.2133 & $\begin{array}{l}249.1086 \\
331.6027 \\
403.1153\end{array}$ & $\begin{array}{l}371.1527 \\
249.0799\end{array}$ & Tricin derivative \\
\hline
\end{tabular}


Table 5. Cont.

\begin{tabular}{|c|c|c|c|c|c|c|c|}
\hline \multirow[b]{2}{*}{ Part } & \multirow[b]{2}{*}{ Comp. * } & \multirow[b]{2}{*}{$\begin{array}{l}\mathrm{tR} \\
(\min )\end{array}$} & \multirow[b]{2}{*}{$\begin{array}{l}\mathrm{UV} \lambda \\
(\mathbf{n m})\end{array}$} & \multicolumn{2}{|l|}{ MS } & \multirow[b]{2}{*}{ MS/MS } & \multirow[b]{2}{*}{$\begin{array}{l}\text { Tentative } \\
\text { Identification }\end{array}$} \\
\hline & & & & {$[\mathbf{M}+\mathbf{H}]^{+}$} & $\begin{array}{l}\text { Main } \\
\text { Fragments }\end{array}$ & & \\
\hline \multirow[b]{2}{*}{$\begin{array}{l}\text { Outer culm } \\
(1 \mathrm{~m})\end{array}$} & 9 & 12.34 & 254,326 & 549.1879 & $\begin{array}{l}197.1128 \\
384.5780 \\
498.6190 \\
\end{array}$ & $\begin{array}{l}447.1168 \\
495.1613\end{array}$ & Not identified \\
\hline & 4 & 13.57 & 254,326 & 639.2403 & $\begin{array}{l}561.3504 \\
215.0037 \\
289.1380 \\
401.0721 \\
485.6067 \\
\end{array}$ & 331.0808 & $\begin{array}{l}O \text {-hexosyl- } O \text { - } \\
\text { deoxyhexosyl } \\
\text { tricin }\end{array}$ \\
\hline $\begin{array}{l}\text { Inner culm } \\
(5 \mathrm{~m})\end{array}$ & 1 & 7.71 & 254,326 & 595.1699 & $\begin{array}{l}401.0721 \\
215.0030 \\
563.2233 \\
379.1075\end{array}$ & $\begin{array}{l}427.0928 \\
457.1007 \\
295.0754 \\
379.0791\end{array}$ & $\begin{array}{l}\text { Di-C,C-hexosyl } \\
\text { apigenin }\end{array}$ \\
\hline \multirow{2}{*}{$\begin{array}{l}\text { Inner culm } \\
(1 \mathrm{~m})\end{array}$} & 10 & 10.69 & 254 & 581.1780 & $\begin{array}{l}401.1565 \\
140.0316 \\
214.9463 \\
284.7471 \\
\end{array}$ & $\begin{array}{l}305.0035 \\
219.0867 \\
131.0860\end{array}$ & Not identified \\
\hline & 11 & 10.92 & 254,326 & 581.2469, & $\begin{array}{l}215.0359 \\
256.0465 \\
329.6107 \\
155.9239 \\
\end{array}$ & 173.9575 & Not identified \\
\hline $\operatorname{Knot}(5 \mathrm{~m})$ & 12 & 7.77 & 254,326 & 597.1854 & $\begin{array}{l}214.9895 \\
197.0173 \\
256.0255 \\
433.8491 \\
\end{array}$ & $\begin{array}{l}149.0515 \\
165.7342 \\
223.7458\end{array}$ & Not identified \\
\hline \multirow[t]{2}{*}{ Knot (1 m) } & 2 & 9.81 & 254,326 & 583.1954 & $\begin{array}{l}249.1038 \\
401.1687 \\
331.5832 \\
237.1147\end{array}$ & $\begin{array}{l}131.0739 \\
232.1788 \\
231.0688\end{array}$ & Tricin derivative \\
\hline & 13 & 17.03 & 254,326 & 441.1956 & $\begin{array}{l}354.2400 \\
212.0524 \\
154.9682 \\
\end{array}$ & $\begin{array}{l}265.1592 \\
177.0511\end{array}$ & Not identified \\
\hline \multirow[t]{2}{*}{ Rhizome } & 14 & 15.16 & 254,326 & 323.1311 & $\begin{array}{l}256.0713 \\
181.0395 \\
196.9698 \\
215.0161 \\
240.9718 \\
\end{array}$ & 169.2761 & Not identified \\
\hline & 15 & 15.92 & 254,326 & 353.1676 & $\begin{array}{l}181.0075 \\
156.0012 \\
255.9951 \\
214.9742\end{array}$ & $\begin{array}{l}177.0655 \\
145.0326 \\
337.1738\end{array}$ & Not identified \\
\hline
\end{tabular}


Table 5. Cont.

\begin{tabular}{|c|c|c|c|c|c|c|c|}
\hline \multirow[b]{2}{*}{ Part } & \multirow[b]{2}{*}{ Comp. * } & \multirow[b]{2}{*}{$\begin{array}{l}\text { tR } \\
(\min )\end{array}$} & \multirow[b]{2}{*}{$\begin{array}{l}\text { UV } \lambda \\
(\mathbf{n m})\end{array}$} & \multicolumn{2}{|l|}{ MS } & \multirow[b]{2}{*}{ MS/MS } & \multirow[b]{2}{*}{$\begin{array}{l}\text { Tentative } \\
\text { Identification }\end{array}$} \\
\hline & & & & {$[\mathbf{M}+\mathbf{H}]^{+}$} & $\begin{array}{l}\text { Main } \\
\text { Fragments }\end{array}$ & & \\
\hline \multirow{20}{*}{ Rhizome } & \multirow{4}{*}{16} & \multirow{4}{*}{16.52} & \multirow{4}{*}{254,326} & \multirow{4}{*}{411.1811} & 215.0015 & 235.1485 & \multirow{4}{*}{ Not identified } \\
\hline & & & & & 206.1046 & 147.0246 & \\
\hline & & & & & 266.0636 & 265.1572 & \\
\hline & & & & & 367.1154 & 177.0882 & \\
\hline & \multirow{4}{*}{13} & \multirow{4}{*}{17.01} & \multirow{4}{*}{254,326} & \multirow{4}{*}{441.2149} & 289.0373 & & \multirow{4}{*}{ Not identified } \\
\hline & & & & & 197.0155 & $\begin{array}{l}263.1526 \\
1770525\end{array}$ & \\
\hline & & & & & 154.0150 & $1 / 7.0535$ & \\
\hline & & & & & 255.9951 & & \\
\hline & \multirow{6}{*}{17} & \multirow{6}{*}{18.43} & \multirow{6}{*}{254,326} & \multirow{6}{*}{455.2147} & 181.0407 & & \multirow{6}{*}{ Not identified } \\
\hline & & & & & 214.9568 & 1720075 & \\
\hline & & & & & 197.0268 & 175.9925 & \\
\hline & & & & & 381.6146 & 124.2000 & \\
\hline & & & & & 266.0714 & & \\
\hline & & & & & 308.6563 & & \\
\hline & \multirow{6}{*}{18} & \multirow{6}{*}{22.73} & & & 214.9897 & & \\
\hline & & & & & 181.0442 & 427.1142 & \\
\hline & & & 254326 & 4451614 & 197.0006 & 177.9142 & Notididified \\
\hline & & & 234,520 & $44 J .1014$ & 317.1962 & 362.6228 & 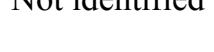 \\
\hline & & & & & 405.2211 & 114.6710 & \\
\hline & & & & & 283.1105 & & \\
\hline & & & & & 196.9985 & 409.1006 & \\
\hline & & & & & 498.0875 & 457.1415 & Di-C,C- \\
\hline & 1 & 7.70 & 254,326 & 595.1482 & 542.1194 & 369.0885 & hexosyl \\
\hline & & & & & 325.0225 & 421.0878 & apigenin \\
\hline & & & & & 249.1168 & 439.1011 & \\
\hline & & & & & 249.1353 & & \\
\hline & & & & & 331.5924 & 207.3230 & \\
\hline & & & & & 605.1899 & 520.5871 & \\
\hline Root t & 2 & 9.90 & 254,326 & 583.2460 & 360.0969 & 286.0984 & Not identified \\
\hline Nove & & & & & 214.9603 & 412.9828 & \\
\hline & & & & & 281.0794 & 388.1342 & \\
\hline & & & & & 403.5934 & & \\
\hline & & & & & & 411.1128 & \\
\hline & & & & & 214.9826 & 429.1186 & \\
\hline & 10 & 1105 & 245326 & 5701288 & 247.0254 & 393.1004 & Notidentified \\
\hline & 17 & $11.0 J$ & $2+J, J 20$ & 519.1200 & 350.0942 & 349.0813 & 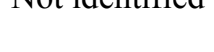 \\
\hline & & & & & 164.0768 & 409.1005 & \\
\hline & & & & & & 295.0939 & \\
\hline
\end{tabular}


Table 5. Cont.

\begin{tabular}{|c|c|c|c|c|c|c|c|}
\hline \multirow[b]{2}{*}{ Part } & \multirow[b]{2}{*}{ Comp. * } & \multirow{2}{*}{$\begin{array}{l}\text { tR } \\
(\min )\end{array}$} & \multirow{2}{*}{$\begin{array}{l}\mathrm{UV} \lambda \\
(\mathbf{n m})\end{array}$} & \multicolumn{2}{|l|}{ MS } & \multirow[b]{2}{*}{ MS/MS } & \multirow{2}{*}{$\begin{array}{l}\text { Tentative } \\
\text { Identification }\end{array}$} \\
\hline & & & & {$[\mathbf{M}+\mathbf{H}]^{+}$} & $\begin{array}{l}\text { Main } \\
\text { Fragments }\end{array}$ & & \\
\hline \multirow{9}{*}{ Root } & \multirow{6}{*}{20} & \multirow{6}{*}{12.37} & \multirow{6}{*}{245,326} & \multirow{6}{*}{549.1083} & 531.1381 & 531.1381 & \multirow{6}{*}{$\begin{array}{l}\text { Di-C-glycosyl } \\
\text { apigenin }\end{array}$} \\
\hline & & & & & 197.0006 & 197.0006 & \\
\hline & & & & & 457.1618 & 457.1618 & \\
\hline & & & & & 337.1038 & 337.1038 & \\
\hline & & & & & 382.8247 & 382.8247 & \\
\hline & & & & & 139.9865 & 139.9865 & \\
\hline & \multirow{3}{*}{5} & \multirow{3}{*}{13.92} & \multirow{3}{*}{245,326} & \multirow{3}{*}{493.1157} & 295.0853 & & \multirow{3}{*}{$\begin{array}{l}O \text {-hexosyl } \\
\text { tricin }\end{array}$} \\
\hline & & & & & 338.5214 & $\begin{array}{l}331.0 / 91 \\
442.3596\end{array}$ & \\
\hline & & & & & $\begin{array}{l}197.0107 \\
475.3166\end{array}$ & 244.4431 & \\
\hline
\end{tabular}

* Compounds that show pseudomolecular ions in mass spectra in both positive and negative ion modes were listed here and indicated in corresponding chromatograms in Figure S1.

Table 6. Partial characterization of water extracts of different parts of bamboo by LCMS-IT-TOF.

\begin{tabular}{|c|c|c|c|c|c|c|c|}
\hline \multirow[b]{2}{*}{ Part } & \multirow[b]{2}{*}{ Comp. * } & \multirow{2}{*}{$\begin{array}{l}\text { tR } \\
(\min )\end{array}$} & \multirow{2}{*}{$\begin{array}{l}\text { UV } \lambda \\
(\mathbf{n m})\end{array}$} & \multicolumn{2}{|l|}{ MS } & \multirow[b]{2}{*}{ MS/MS } & \multirow{2}{*}{$\begin{array}{l}\text { Tentative } \\
\text { Identification }\end{array}$} \\
\hline & & & & {$[\mathbf{M}+\mathbf{H}]^{+}$} & $\begin{array}{l}\text { Main } \\
\text { Fragments }\end{array}$ & & \\
\hline \multirow{20}{*}{ Leaf } & \multirow{4}{*}{21} & \multirow{4}{*}{8.36} & \multirow{4}{*}{254,278} & \multirow{4}{*}{346.0437} & 206.9890 & 145.0514 & \multirow{4}{*}{ Not identified } \\
\hline & & & & & 235.0134 & 292.1701 & \\
\hline & & & & & 173.0079 & 177.0293 & \\
\hline & & & & & 242.0372 & 313.7123 & \\
\hline & \multirow{5}{*}{22} & \multirow{5}{*}{12.69} & \multirow{5}{*}{254,278} & \multirow{5}{*}{355.0828} & 207.0098 & \multirow{5}{*}{$\begin{array}{l}174.9783 \\
163.2186\end{array}$} & \multirow{5}{*}{$\begin{array}{l}\text { Chlorogenic } \\
\text { acid }\end{array}$} \\
\hline & & & & & 146.9837 & & \\
\hline & & & & & 275.0457 & & \\
\hline & & & & & 235.0089 & & \\
\hline & & & & & 185.1625 & & \\
\hline & \multirow{6}{*}{23} & \multirow{6}{*}{19.19} & \multirow{6}{*}{254,278} & \multirow{6}{*}{449.1190} & 207.0091 & \multirow{6}{*}{$\begin{array}{l}299.0534 \\
353.0652 \\
383.0690 \\
339.0555 \\
395.0833\end{array}$} & \multirow{6}{*}{$\begin{array}{l}\text { 8-C-glucosyl } \\
\text { luteolin } \\
\text { (orientin) }\end{array}$} \\
\hline & & & & & 234.9587 & & \\
\hline & & & & & 243.0011 & & \\
\hline & & & & & 177.0516 & & \\
\hline & & & & & 285.0846 & & \\
\hline & & & & & 377.4037 & & \\
\hline & \multirow{5}{*}{24} & \multirow{5}{*}{19.79} & \multirow{5}{*}{254,278} & \multirow{5}{*}{449.0907} & & 299.0532, & \multirow{5}{*}{$\begin{array}{l}\text { 6- } C \text {-glucosyl } \\
\text { luteolin } \\
\text { (isoorientin) }\end{array}$} \\
\hline & & & & & 431.0489 & 353.0659, & \\
\hline & & & & & 301.1575 & 395.0849, & \\
\hline & & & & & 215.0161 & 329.0699 & \\
\hline & & & & & & 383.0960 & \\
\hline
\end{tabular}


Table 6. Cont.

\begin{tabular}{|c|c|c|c|c|c|c|c|}
\hline \multirow[b]{2}{*}{ Part } & \multirow[b]{2}{*}{ Comp. * } & \multirow{2}{*}{$\begin{array}{l}\text { tR } \\
(\min )\end{array}$} & \multirow{2}{*}{$\begin{array}{l}\text { UV } \lambda \\
(\mathbf{n m})\end{array}$} & \multicolumn{2}{|l|}{ MS } & \multirow[b]{2}{*}{ MS/MS } & \multirow{2}{*}{$\begin{array}{l}\text { Tentative } \\
\text { Identification }\end{array}$} \\
\hline & & & & {$[\mathbf{M}+\mathbf{H}]^{+}$} & $\begin{array}{l}\text { Main } \\
\text { Fragments }\end{array}$ & & \\
\hline \multirow{17}{*}{ Leaf } & \multirow{5}{*}{25} & \multirow{5}{*}{20.86} & \multirow{5}{*}{254,278} & \multirow{5}{*}{433.1147} & 206.9915 & & \multirow{5}{*}{$\begin{array}{l}\text { 8- } C \text {-glucosyl } \\
\text { apigenin } \\
\text { (vitexin) }\end{array}$} \\
\hline & & & & & 234.0091 & 177.7364 & \\
\hline & & & & & 174.9798 & 245.2327 & \\
\hline & & & & & 251.1581 & 100.8017 & \\
\hline & & & & & 279.0238 & & \\
\hline & \multirow{3}{*}{6} & \multirow{3}{*}{22.32} & \multirow{3}{*}{254,278} & \multirow{3}{*}{433.1255} & 175.0077 & & \multirow{3}{*}{$\begin{array}{l}\text { 6- } C \text {-glucosyl } \\
\text { apigenin } \\
\text { (isoviterxin) }\end{array}$} \\
\hline & & & & & 313.0549 & 168.5285 & \\
\hline & & & & & 455.0990 & & \\
\hline & \multirow{4}{*}{5} & \multirow{4}{*}{25.95} & \multirow{4}{*}{254,278} & \multirow{4}{*}{493.1481} & 206.9978 & & \multirow{4}{*}{$\begin{array}{l}O \text {-hexosyl } \\
\text { tricin }\end{array}$} \\
\hline & & & & & 371.0754 & 3210757 & \\
\hline & & & & & 159.0127 & ונדוס. & \\
\hline & & & & & 351.1284 & & \\
\hline & & & & & 191.0127 & & \\
\hline & & & & & 207.0049 & & $O$-hexosyl- $O-$ \\
\hline & 4 & 26.75 & 254,278 & 639.1185 & 235.0432 & 3150479 & deoxyhexosyl \\
\hline & & & & & 253.1692 & & \\
\hline & & & & & 460.8544 & & \\
\hline & & & & & 206.9941 & & \\
\hline & 21 & 857 & 254278 & 3460656 & 234.9992 & 2482391 & Not identified \\
\hline & & & & & 174.9993 & & 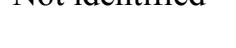 \\
\hline & & & & & 191.0522 & & \\
\hline Branch & & & & & 579.1455 & & \\
\hline Didnct & & & & & 371.1160 & $\begin{array}{l}451.1221 \\
3250685\end{array}$ & \\
\hline & 1 & 1602 & 254278 & 5051387 & 249.1300 & $\begin{array}{l}325.0685 \\
427.1048\end{array}$ & Di- $C, C$-hexosyl \\
\hline & 1 & 10.93 & 234,218 & $595.138 /$ & 311.0476 & $\begin{array}{l}421.1048 \\
3790890\end{array}$ & apigenin \\
\hline & & & & & 235.0080 & 2950745 & \\
\hline & & & & & 207.0031 & & \\
\hline & & & & & 206.9909 & & \\
\hline & & & & & 235.0080 & 152.0649 & \\
\hline & 21 & 8.65 & 254,278 & 346.0495 & 158.9887 & 257.2977 & Not identified \\
\hline & & & & & 174.9798 & 172.7825 & \\
\hline & & & & & 193.0579 & & \\
\hline & & & & & 579.2583 & & \\
\hline Outer & & & & & 457.1415 & 457.1184 & \\
\hline culm & 1 & 16.90 & 254,278 & 595.1733 & 371.0852 & 427.1094 & Di-C,C-hexosyl \\
\hline$(5 \mathrm{~m})$ & & & & & 249.1032 & 325.0682 & ap1genın \\
\hline & & & & & 206.9530 & & \\
\hline & & & & & 557.1886 & & \\
\hline & & & & & 441.1388 & $\begin{array}{l}331.0 / 88 \\
3150678\end{array}$ & $O$-hexosyl- $O-$ \\
\hline & 4 & 26.62 & 254,278 & 639.1777 & 355.1725 & 51 & deoxyhexosyl \\
\hline & & & & & 175.0428 & 210.0594 & tricin \\
\hline & & & & & 159.0067 & & \\
\hline
\end{tabular}


Table 6. Cont.

\begin{tabular}{|c|c|c|c|c|c|c|c|}
\hline Part & Comp. * & $\begin{array}{l}\text { tR } \\
(\min )\end{array}$ & $\begin{array}{l}\text { UV } \lambda \\
(\mathbf{n m})\end{array}$ & $\begin{array}{l}\text { MS } \\
{[\mathbf{M}+\mathbf{H}]^{+}}\end{array}$ & $\begin{array}{l}\text { Main } \\
\text { Fragments }\end{array}$ & MS/MS & $\begin{array}{l}\text { Tentative } \\
\text { Identification }\end{array}$ \\
\hline \multirow{12}{*}{$\begin{array}{l}\text { Outer } \\
\text { culm } \\
(1 \mathrm{~m})\end{array}$} & \multirow{3}{*}{21} & \multirow{3}{*}{8.59} & \multirow{3}{*}{254,278} & \multirow{3}{*}{346.0550} & 207.0078 & & \multirow{3}{*}{ Not identified } \\
\hline & & & & & 174.9546 & 152.0448 & \\
\hline & & & & & 235.0164 & 202.7962 & \\
\hline & \multirow{5}{*}{1} & \multirow{5}{*}{16.89} & \multirow{5}{*}{254,278} & \multirow{5}{*}{595.1143} & $\begin{array}{l}210.050 / \\
579.1475\end{array}$ & & \multirow{5}{*}{$\begin{array}{l}\mathrm{Di}-C, C \text {-hexosyl } \\
\text { apigenin }\end{array}$} \\
\hline & & & & & 249.1167 & 427.0888 & \\
\hline & & & & & 371.1295 & 379.0915 & \\
\hline & & & & & 207.0176 & 295.0598 & \\
\hline & & & & & 175.0050 & & \\
\hline & \multirow{4}{*}{26} & \multirow{4}{*}{25.86} & \multirow{4}{*}{254,278} & \multirow{4}{*}{295.0849} & 207.0089 & & \multirow{4}{*}{ Not identified } \\
\hline & & & & & 219.0139 & 135.8380 & \\
\hline & & & & & 174.9589 & 178.2954 & \\
\hline & & & & & 147.0010 & & \\
\hline $\begin{array}{l}\text { Inner } \\
\text { culm } \\
(5 \mathrm{~m}) \\
\end{array}$ & 27 & 16.95 & 254,278 & 165.0803 & 146.9779 & - & $p$-courmaric acid \\
\hline Inner & \multirow[b]{2}{*}{27} & \multirow[b]{2}{*}{16.88} & \multirow[b]{2}{*}{254,278} & \multirow[b]{2}{*}{165.0865} & \multirow[b]{2}{*}{146.9779} & \multirow[b]{2}{*}{-} & \multirow[b]{2}{*}{$p$-courmaric acid } \\
\hline $\begin{array}{l}\text { culm } \\
(1 \mathrm{~m})\end{array}$ & & & & & & & \\
\hline $\begin{array}{l}\text { Knot } \\
(5 \mathrm{~m})\end{array}$ & 27 & 17.06 & 254,278 & 165.0806 & 146.9779 & - & $p$-courmaric acid \\
\hline $\begin{array}{l}\text { Knot } \\
(1 \mathrm{~m})\end{array}$ & 27 & 16.89 & 254,278 & 165.2503 & 147.0356 & - & $p$-courmaric acid \\
\hline \multirow{10}{*}{ Rhizome } & & & & & 206.9530 & & \\
\hline & 28 & 705 & 254278 & 3200130 & 234.9505 & 2508027 & Not identified \\
\hline & 20 & 1.00 & (2) & 300.0150 & 174.9735 & & 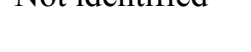 \\
\hline & & & & & 266.0403 & & \\
\hline & & & & & 206.9626 & & \\
\hline & 21 & 8.66 & 254.278 & 346.0354 & 233.9144 & 152.0740 & Not identified \\
\hline & & & & & 174.9779 & 174.0595 & \\
\hline & & & & & 214.9463 & & \\
\hline & 27 & 1602 & 254278 & 1653803 & 146.9779 & 1328580 & $n_{-}$courmaric a \\
\hline & & 10.02 & (2) & (10000 & 159.0007 & & \\
\hline Root & 27 & 16.91 & 254.278 & 165.0853 & 146.9894 & - & $p$-courmaric acid \\
\hline & & & & & 159.0127 & & \\
\hline
\end{tabular}

* Compounds that show pseudomolecular ions in mass spectra in both positive and negative ion modes were listed here and indicated in corresponding chromatograms in Figure S2.

Immunoglobulin E (IgE) is well known as a trigger of allergic reactions [21]. Here, the level of $\operatorname{IgE}$ production in Peripheral Blood Lymphocytes (PBL) was used to evaluate anti-allergy activity of the extracts from different parts of bamboo. 
Table 4 shows the anti-allergy activity of the ethanol extracts and the hot water extracts of $P$. pubescens. Compared with the IgE concentration of controls, hot water extracts of leaves, outer culm $(5 \mathrm{~m}, 1 \mathrm{~m})$, inner culm $(5 \mathrm{~m})$, knots $(5 \mathrm{~m})$, and rhizomes significantly inhibited the production of $\mathrm{IgE}$ in PBL. Among these extracts, leaves showed the strongest anti-allergy activity with an inhibition rate of $42.8 \%$. The inhibition rates of other extracts were $29.3 \%$ (outer culm at $5 \mathrm{~m}$ ), $35.9 \%$ (outer culm at $1 \mathrm{~m}$ ), $35.9 \%$ (inner culm at $5 \mathrm{~m}$ ), $35.9 \%$ (knots at $5 \mathrm{~m}$ ) and 37.2\% (rhizomes), respectively. On the other hand, ethanol extracts showed no effect on IgE production in PBL.

\subsection{Phytochemical Profile}

The chromatographic profiles of each extracts were determined through LCMS analysis (Figures S1 and S2). Very different chromatograms can be seen for the extracts from the leaf, branch, outer culm, inner culm, knot rhizome or root parts, suggesting the ethanol extracts and water extracts from different parts of bamboo have very different chemical compositions. Based on the data from both positive and negative MS and MS/MS spectra, the component of each extract was partially identified referring to the standards or the literature [22,23]. For example, di- $C, C$-hexosylapigenin (compound 1) was first identified in the ethanol extract of outer culm $(1 \mathrm{~m})$ for the presence of a pseudomolecular ion at $m / z 595[\mathrm{M}+\mathrm{H}]^{+}$and four typical fragment ions of di-C, $C$-hexosyl-flavones [22,24]. They are $\mathrm{m} / \mathrm{z}$ $325[(\mathrm{M}+\mathrm{H})-120-150]^{+}, \mathrm{m} / \mathrm{z} 427[(\mathrm{M}+\mathrm{H})-150-18]^{+}, \mathrm{m} / \mathrm{z} 457[(\mathrm{M}+\mathrm{H})-120-18]^{+}$and m/z $379[(\mathrm{M}+\mathrm{H})-$ 120-96 $]^{+}$(Figure S3A). In other extracts, di-C,C-hexosyl apigenin was identified through the pseudomolecular ion, the typical fragment ions and the retention time referring to that in the ethanol extract of outer culm $(1 \mathrm{~m})$. Similarly, $O$-hexosyl- $O$-deoxyhexosyl tricin (compound 4) was tentatively identified because the presence of a pseudomolecular ion at $m / z 639[\mathrm{M}+\mathrm{H}]^{+}$, the characteristic fragment ion for $O$-hexosyl-O-deoxyhexosyl derivatives at $m / z 331$ [(M+H)-162-146] $]^{+}$[18], fragment ion at $\mathrm{m} / \mathrm{z} 561[(\mathrm{M}+\mathrm{H})-60-18]^{+}$and $357[(\mathrm{M}+\mathrm{H})-120-162]^{+}$(Figure S3B). 6-C-Glucosylapigenin (compound 6) was mainly identified based on the appearance of pseudomolecular ion at $\mathrm{m} / \mathrm{z} 433$ $[\mathrm{M}+\mathrm{H}]^{+}$and typical mono- $C$-glycoside fragment ions at $m / z 313[(\mathrm{M}+\mathrm{H})-120]^{+}, m / z 283[(\mathrm{M}+\mathrm{H})-150]^{+}$ and $m / z 337[(\mathrm{M}+\mathrm{H})-60-18-18]^{+}$. The position of the mono- $C$-glycosylation was indicated by the appearance of fragment at $m / z 341[(\mathrm{M}-\mathrm{H})-90]^{-}$and $m / z 323$ [(M-H)-90-18] $]^{-}$[23,25] (Figure S3C). Chlorogenic acid (compound 22) and $p$-courmaric acid (compound 27) were identified by their identical retention times, pseudomolecular ions and fragment ions as the corresponding standard compounds. Chlorogenic acid showed a clear psudomolecular at $m / z 353[\mathrm{M}-\mathrm{H}]^{-}$and a dominant fragment ion at $m / z \quad 191$ [(M-H)-162] $]^{-}$, while $p$-courmaric acid showed a clear psudomolecular at $m / z 163[\mathrm{M}-\mathrm{H}]^{-}$. Because the complex composition of the extracts, only the fractions showing pseudomolecular ions in both positive and negative ion modes were listed in the table and tentatively identified (Tables 5 and 6). These fractions were indicated in corresponding chromatograms (Figure S1 and S2), functioning as the markers in the characteristic chromatogram of each extract. 
The results showed that the glycoside, di- $C, C$-hexosylapigenin, which existed in the ethanol extracts of leaf, outer culm, inner culm, root and water extracts of leaf and branch (Tables 5 and 6), is the most common compound in the different parts of bamboo. Besides di- $C, C$-hexosylapigenin, three other apigenin derivatives, 6- $C$-glucosylapigenin (compound 6), 8-C-glucosylapigenin (compound 25) and di- $C$-glycosylapigenin (compound 20) were also found in different extracts. 6- $C$-Glucosylapigenin was found in the ethanol extract of branch and water extract of leaf, while 8 - $C$-glucosylapigenin was only found in the water extract of leaf and di- $C$-glycosylapigenin was found in the ethanol extract of root. Another major component found in these extracts was tricin derivatives. $O$-Hexosyl- $O$ deoxyhexosyl tricin (compound 4) was found in both ethanol extract and water extract of leaf and outer culm, while $O$-hexosyltricin (compound 5) was found in the ethanol extracts of leaf and root and the water extract of leaf. Two luteolin derivatives, 6- $C$-glucosylluteolin (compound 24) and 8- $C$ glucosylluteolin (compound 23) were also found in the water extract of leaf. In the water extract of outer culm, inner culm, rhizome and root, $p$-courmaric acid (compound 27) was found.

Although the components of each extract were only partially identified and a quantitative analysis was not done, we tried to find some hints indicating possible active compounds by comparing the results from the LC-MS and activity assays. Apigenin is a naturally occurring flavonoid, which has been reported to possess various activities, including antioxidation [26], antimutagenic [27], antiinflammation [28], and anticarcinogenic activities [29], and so on. Its derivatives 6-C-glucosylapigenin (isovitexin, compound 6) and 8-C-glucosylapigenin (vitexin, compound 25) were found to have anti-diabetic complication activity and anti-Alzheimer's disease activity [30]. Here, 8- $C$ glucosylapigenin (compound 25) was only found in the water extract of leaf that showed the strongest anti-allergy activity among all extracts (Table 4), suggesting 8-C-glucosylapigenin had the higher possibility than other three apigenin derivatives to be responsible for the anti-allergy activity. In addition, 8-C-glucosylluteolin (orientin, compound 23), 6-C-glucosylluteolin (isoorientin, compound 24) and chlorogenic acid (compound 22) were also only found in the water extract of leaf (Table 6). Luteolin and luteolin 7-glucoside had been reported to show allergy-preventive activity [31,32]. Chlorogenic acid had a series of biological effects [33] and also had been found to have allergy-preventive activity [34]. Therefore, the most probable compounds responsible for the anti-allergy of bamboo were 8-Cglucosylapigenin, the luteolin derivatives and chlorogenic acid (compound 22). $O$-Hexosyl-Odeoxyhexosyl tricin (compound 4) mainly appeared in the ethanol extract of outer culm that showed strongest antibacterial and melanin inhibition activity (Tables 3 and 1), suggesting $O$-hexosyl- $O$ deoxyhexosyl tricin was possibly the compound responsible for the antibacterial and melanin inhibition activity, although tricin had no activity against $S$. aureus [35]. The ethanol extract of inner culm and branch showed best antioxidant activity (Table 2), but we couldn't identify more compounds from them so far except for 6- $C$-glucosylapigenin (compound 6) and di-C,C-hexosyl apigenin (compound 1). Apigenin was already known as an antioxidant [36,37]. Further studies are needed to find out the exact active compounds responsible for these bioactivities of bamboo. 


\section{Experimental}

\subsection{Plant Materials}

Whole plants of 1 or 2-year old $P$. pubescens were harvested at Kurume, Fukuoka Prefecture, Japan. The average height of the harvested bamboo was $14 \mathrm{~m}$. Then, plants were separated into the following parts: leaves, branches, outer culm, inner culm, knots, rhizomes and roots (Figure 1). At that time, the outer culm, inner culm and knots were obtained separately from heights of $5.0 \pm 0.3$ and $1.0 \pm 0.3 \mathrm{~m}$ above ground level. Each part was freeze-dried and milled into powder.

Milled freeze-dried $P$. pubescens samples were extracted with $99.5 \%$ ethanol at room temperature with a shaker at $200 \mathrm{rpm}$ for $48 \mathrm{~h}$ and then filtered. The ethanol extracts were concentrated by a rotary evaporator. The yields of ethanol extracts against each dried powder were as follows: leaves, 4.84\%; branches, $1.08 \%$; outer culm $(5 \mathrm{~m}), 4.56 \%$; outer culm $(1 \mathrm{~m}), 4.69 \%$; inner culm $(5 \mathrm{~m}), 0.27 \%$; inner culm $(1 \mathrm{~m}), 0.32 \%$; knots $(5 \mathrm{~m}), 1.47 \%$; knots $(1 \mathrm{~m}), 1.55 \%$; rhizomes, $0.45 \%$ and roots, $2.63 \%$. To prepare the hot water extracts, $P$. pubescens samples were extracted with hot water at $120^{\circ} \mathrm{C}$ for $20 \mathrm{~min}$ and the extracted solutions were freeze dried. The yields of hot water extracts were as follows: leaves, 10.4\%; branches, $2.67 \%$; outer culm (5 m), 2.96\%; outer culm ( $1 \mathrm{~m}), 3.69 \%$; inner culm ( $5 \mathrm{~m}), 1.60 \%$; inner culm (1 m), 2.21\%; knots (5 m), 3.64\%; knots ( $1 \mathrm{~m}), 4.94 \%$; rhizomes, $2.49 \%$ and roots $3.25 \%$.

\subsection{Melanin Biosynthesis Assay}

This assay was performed as previously described by Arung et al. [38]. The B16 melanoma cells were maintained in EMEM supplemented with $10 \%(\mathrm{v} / \mathrm{v})$ fetal bovine serum (FBS) and $0.09 \mathrm{mg} / \mathrm{mL}$ theophylline. Cells were incubated at $37{ }^{\circ} \mathrm{C}$ in a humidified atmosphere of $5 \% \mathrm{CO}_{2}$. Cells were placed into a 24-well plate at a density of $1 \times 10^{5}$ cells $/ \mathrm{mL}$ and incubated for $24 \mathrm{~h}$ in medium prior to treatment with extract. After $24 \mathrm{~h}$, the medium was replaced with $998 \mu \mathrm{L}$ of fresh medium, and $2 \mu \mathrm{L}$ of ethanol extract dissolved in dimethylsulfoxide (DMSO) or hot water extract dissolved in sterilized water was added. The cells were incubated for an additional $48 \mathrm{~h}$; then the medium was replaced with fresh medium and extract was added again. After $24 \mathrm{~h}$, the remaining adherent cells were used to determine the melanin content and cell viability (see below). To find possible candidates for whitening or tanning agents, we classified the tested extracts into three types (Type A, B, and C). Samples which showed a percentage of melanin content equal to or lower than $20 \%$ of cell viability (e.g., CV-MC $\geq$ 20) were judged as possible whitening agents, and classified as type A. In the other hand, samples which showed a percentage of melanin content equal to or higher than $20 \%$ of cell viability (e.g., $\mathrm{MC}-\mathrm{CV} \geq 20$ ) were judged as possible tanning agents, and classified as type B. Finally, samples showed a percentage of cell viability equal to or lower than $90 \%$ were judged to be cytotoxic and classified as type $\mathrm{C}$.

\subsubsection{Cell Viability}

Cell viability (CV) was determined by use of the microculture tetrazolium technique (MTT) [38]. Culture was initiated, and after incubation, $50 \mu \mathrm{L}$ of MTT [3-(4,5-dimethylthiazol-2-yl)-2,5diphenyltetrazolium bromide] in phosphate buffered saline $(5 \mathrm{mg} / \mathrm{mL})$ was added to each well. The 
plates were incubated for $4 \mathrm{~h}$. After removing the medium, formazan crystals were dissolved in $1.0 \mathrm{~mL}$ of $0.04 \mathrm{M} \mathrm{HCl}$ in isopropanol and the absorbance was measured at $570 \mathrm{~nm}$ relative to $630 \mathrm{~nm}$.

\subsubsection{Determination of Melanin Content}

The melanin content (MC) of cells after treatment with the extract was determined as follows. After removing the medium and washing the cells, the cell pellet was dissolved in $1.0 \mathrm{~mL}$ of $1 \mathrm{M} \mathrm{NaOH}$. The crude cell extracts were assayed using a microplate reader (Bio-Tek, Winooski, VT, USA) at 405 $\mathrm{nm}$ to determine the melanin content. The results from the samples were analyzed as a percentage of the control culture. Arbutin was used as a positive control.

\subsection{Antioxidant Assays}

\subsubsection{Oxygen Radical Absorbance Capacity Assay}

The oxygen radical absorbance capacity (ORAC) assay was performed as described previously by Prior et al. [39]. Data are expressed as milligrams of Trolox equivalent (TE) per milligram of sample extract (mg TE/mg).

\subsubsection{Superoxide Dismutase-Like Activity}

Superoxide dismutase (SOD)-like activity was evaluated using the SOD Assay Kit-WST (Dojindo Molecular Technologies, Kumamoto, Japan) according to the method described in previous studies [40]. Sample were dissolved in water or ethanol and added to the WST working solutions $(200 \mu \mathrm{L})$ containing 2-(4-iodophenyl)-3-(4-nitrophenyl)-5-(2, 4-disulfophenyl)-2- $H$-tetrazolium in $50 \mathrm{mM}$ carbonate buffer ( $\mathrm{pH}$ 10.2). An enzyme working solution $(20 \mu \mathrm{L})$ containing xanthine oxidase in the same buffer was added and then incubated for $10 \mathrm{~min}$. The absorbance of each sample was measured at $450 \mathrm{~nm}$ in a Tecan Spectra microplate reader (Tecan Japan, Kanagawa, Japan). One unit of SOD-like activity was defined as the amount of extract in $20 \mu \mathrm{L}$ of sample solution that inhibits the reduction reaction of WST-1 with superoxide anions by 50\%. The SOD-like activity (U/mg) of each extract was calculated using the $50 \%$ inhibition value $\left(\mathrm{IC}_{50}\right)$ of the extract.

\subsubsection{ABTS Radical Cation Decolorization Assay}

The ABTS assay was mostly based on the methods described by Re et al. [41] in which ABTS ${ }^{+}$, the oxidant, was generated by persulfate oxidation of ABTS [2,2'-azinobis (3-ethylbenzothiazoline-6sulfonic acid)]. Specifically, to $5 \mathrm{~mL}$ of $7 \mathrm{mM} \mathrm{ABTS}$ ammonium aqueous solution, $88 \mu \mathrm{L}$ of $140 \mathrm{mM}$ potassium peroxydisulfate $\left(\mathrm{K}_{2} \mathrm{~S}_{2} \mathrm{O}_{8}\right)$ was added, and the resulting mixture was then allowed to stand at room temperature for $12-16 \mathrm{~h}$, yielding a dark blue solution. The mixture was then adjusted by $99.5 \%$ ethanol so that it gave an absorbance of $0.7 \pm 0.02$ units at $734 \mathrm{~nm}$ (UVmini-1240, Shimadzu, Kyoto, Japan) to make the working solution. One milliliter of working solution was mixed with $10 \mu \mathrm{L}$ of extract dissolved in ethanol and shaken well for $10 \mathrm{~s}$; after 4 min of incubation at $30^{\circ} \mathrm{C}$, the absorbance of the reaction mixture was measured at $734 \mathrm{~nm}$. 


\subsection{Antibacterial Assay}

The antibacterial assay was mostly based on the methods described by Tanaka et al. [10]. S. aureus (NBRC 1273) was used for the antibacterial assay. A single colony of the test strain was taken and $5 \mathrm{~mL}$ of nutrient broth medium was added to it. This culture was incubated at $37{ }^{\circ} \mathrm{C} \pm 1{ }^{\circ} \mathrm{C}, 120 \mathrm{rpm}$ for $20 \mathrm{~h}$. It was then added to the bacterial suspension to prepare a bacterial concentration at $10^{5} \mathrm{CFU} / \mathrm{mL}$. The bacterial solution was used for the subsequent antibacterial assay. Each sample was dissolved in DMSO for ethanol extract or sterilized water for hot water extract at maximum concentration. Into each well of a 96-well plate were added $133.5 \mu \mathrm{L}$ of NB medium, $15 \mu \mathrm{L}$ of bacteria suspension, and $1.5 \mu \mathrm{L}$ of solvent with or without each sample. Also, sorbic acid was used as a positive control. The plate was incubated at $37^{\circ} \mathrm{C} \pm 1{ }^{\circ} \mathrm{C}, 1160 \mathrm{rpm}$ for $18 \mathrm{~h}$. Finally, bacterial growth was measured by a microplate reader at $630 \mathrm{~nm}$ (Biotek-ELX800, BioTek). The minimum inhibitory concentration (MIC) is the lowest concentration of an antibacterial agent required to completely inhibit the growth of a particular bacteria, while the minimum bactericidal concentration (MBC) is the lowest concentration of an antibacterial agent required to kill the bacteria. Here, the MIC of active extracts was determined through the antibacterial assay using gradient concentrations. And MBC of them were further determined as follows: a $20 \mu \mathrm{L}$ aliquot was taken from the wells that treated with extract at higher concentration than its MIC and mixed with180 $\mu \mathrm{L}$ of fresh medium. Then, $100 \mu \mathrm{L}$ of the mixture was used to do the subculture on nutrient agar plate. After $24 \mathrm{~h}$ incubation at $37{ }^{\circ} \mathrm{C} \pm 1{ }^{\circ} \mathrm{C}$, the colony formation was evaluated. The minimum concentration that leaded to no colony growing on the agar plate was considered as the MBC.

\subsection{Immunoglobulin E (IgE) Production Assay}

Peripheral blood lymphocytes (PBL) were first separated from heparinized blood of healthy donors using Ficoll-Paque Plus (GE Healthcare, Uppsala, Sweden). And then, PBL cells were cultured in ERDF medium (Kyokuto Pharmaceuticals, Tokyo, Japan) supplemented with 5\% FBS, 10\% human plasma, $10 \mathrm{ng} / \mathrm{mL}$ of recombinant human IL-4 and IL-6 (R\&D Systems, USA), $10 \mu \mathrm{g} / \mathrm{mL}$ of muramyl dipeptide (MDP) (Sigma, St.Louis, MO, USA) and $100 \mathrm{ng} / \mathrm{mL}$ of the cedar pollen antigen Cry j 1 (Hayashibara Biochemical Laboratories, Okayama, Japan) at the density of $2.0 \times 10^{6}$ cells $/ \mathrm{mL} .198 \mu \mathrm{L}$ of such cell suspension and $2 \mu \mathrm{L}$ of $6 \mathrm{mg} / \mathrm{ml}$ extract in $10 \%$ DMSO solution were added into 96 -well plates (final concentration of extract was $60 \mu \mathrm{g} / \mathrm{mL}$ ). The plate was incubated in a humidified $37^{\circ} \mathrm{C}, 5 \%$ $\mathrm{CO}_{2}$ incubator for 10 days. The total $\mathrm{IgE}$ concentration in the supernatant was measured by sandwich ELISA (enzyme-linked immunosorbent assay). Briefly, 96-well microplates were coated with anti-human IgE antibody (Biosource, Camarillo, CA, USA). The antibody-coated wells were blocked with $1.0 \%$ BSA, following by adding the samples. After washing with PBS containing $0.05 \%$ of Tween 20 for three times, biotin-conjugated antihuman $\operatorname{IgE}$ antibody (Biosource) and horseradish peroxidase-conjugated streptavidin were added. Finally, a substrate solution $[0.1 \mathrm{M}$ citrate buffer (pH 4.0) containing $0.003 \%$ of $\mathrm{H}_{2} \mathrm{O}_{2}$ and $0.3 \mathrm{mg} / \mathrm{mL} p$-2,2'-azino-bis (3-ethylbenzothiazoline-6sulfonic acid) diammonium salt] was added. After $15 \mathrm{~min}$, the absorbance was measured at 414 and $490 \mathrm{~nm}$ by the microplate reader (iMark, Bio-Rad, Hercules, CA, USA). The relative IgE production was calculated according to the absorbance at $414 \mathrm{~nm}$ and $490 \mathrm{~nm}$, and the final inhibition rate was 
calculated using the following formula: Inhibition rate $(\%)=(1-\operatorname{IgE}$ production in treated cells/IgE production in control cells) $\times 100$.

\subsection{LCMS Analysis}

All extracts were subjected to LCMS analysis using a high-speed liquid chromatography mass spectrometry that combines with iron-trap and time-of-flight technologies (LCMS-IT-TOF, Shimadzu, Tokyo, Japan). The instrument was fitted with an Inertsil ODS-3, $5 \mu \mathrm{m}, 1.5 \times 150 \mathrm{~mm}$ column (GL Science, Tokyo, Japan). The oven temperature was set at $40{ }^{\circ} \mathrm{C}$. A mobile phase composed of solvent A $(0.3 \%$ acetic acid in water) and B (0.3\% acetic acid in acetonitrile or methanol) was employed for the separation. Acetonitrile was used in solvent B for the analysis of ethanol extract, while methanol was used for water extract. The mobile phase was consecutively programmed as follows: $0 \sim 60 \mathrm{~min}, \mathrm{~A}$ $90 \sim 0 \%$, B 10\% 100\%; 60 65 min, A 0, B 100\%; 65 66 min, A 0\% 90\%, B 100\% 10\%; a $10 \mathrm{~min}$ post-run was used after each analysis. The total flow rate was $0.15 \mathrm{~mL} / \mathrm{min}$. Basing on the previous result of HPLC-PDA analysis, the LC chromatograms of ethanol extracts and water extracts were obtained at UV $254 \mathrm{~nm}, 326 \mathrm{~nm}$ and 254, $278 \mathrm{~nm}$, respectively. The MS instrument was operated using an ESI source in both positive and negative ionization mode with survey scans acquired from $\mathrm{m} / z 100$ to 1000 for both MS and MS/MS. Ionization parameters were as follows: probe voltage, $\pm 4.5 \mathrm{kV}$; nebulizer gas flow, $1.5 \mathrm{~L} / \mathrm{min}$; CDL temperature, $200{ }^{\circ} \mathrm{C}$; heat block temperature, $200{ }^{\circ} \mathrm{C}$.

The samples were dissolved with initial mobile phase $(1 \mathrm{mg} / \mathrm{mL})$ and filtered through a $0.45-\mu \mathrm{m}$ filter. A volume of $5 \mu \mathrm{L}$ of each sample was injected for the analysis. 8 compounds that had been found in different bamboo species were analyzed and used as a standard. They were catechin (Sigma-Aldrich, Munich, Germany), caffeic acid (Tokyo Chemical Industry, Tokyo, Japan), syringic acid (Tokyo Chemical Industry), chlorogenic acid (Sigma-Aldrich), $p$-courmaric acid (Sigma-Aldrich), rutin (Wako, Tokyo, Japan), trans-ferulic acid (Tokyo Chemical Industry) and luteolin-7-O-glucoside (EXTRASYNTHESE, Genay, France).

\section{Conclusions}

In this study, the effect of ethanol and hot water extracts of various parts of bamboo on the melanin biosynthesis regulation (inhibition or stimulation), antioxidation, antibacterial and anti-allergy were comparatively evaluated. We found that the extracts showed different bioactivities in different degrees. For the melanin biosynthesis inhibition, the hot water extracts of outer culm $(5 \mathrm{~m})$ and rhizome showed the best activities. For the melanin biosynthesis stimulation, the ethanol extract of inner culm $(1 \mathrm{~m})$ showed the strongest activity. For the antioxidant activity, the ethanol extracts of inner culm $(1 \mathrm{~m})$, branch and inner culm $(5 \mathrm{~m})$ showed the strongest activities. For antibacterial activity against $S$. aureus, the ethanol extracts of outer culm $(5 \mathrm{~m}$ and $1 \mathrm{~m})$ showed the strongest activities. The MIC and $\mathrm{MBC}$ for both extracts were 400 and $1600 \mu \mathrm{g} / \mathrm{mL}$, respectively. For anti-allergy activity, the water extract of leaf showed the best IgE inhibition effect. Extracts from the outer culm and inner culm were found to be the most active extracts.

Different parts of bamboo showed different bioactivities, which also varied with the extraction solvent. The difference in chromatographic profile and identified component to some extent explained the different bioactivities of these extracts. The most possible compounds responsible for anti-allergy 
activity of this bamboo were 8-C-glucosyl apigenin, luteolin derivatives and chlorogenic acid. $O$-hexosyl- $O$-deoxyhexosyl tricin was the possible compound responsible for the antibacterial and melanin inhibition activity of bamboo, while apigenin derivatives might be the compounds responsible for the antioxidant activity. This information would be helpful for the further research on the active compounds in bamboo. Taken together, our study provides valuable data to support that bamboo has great potential to be used in the cosmetic industry as well as other health-related industry.

\section{Supplementary Materials}

Supplementary materials can be accessed at: http://www.mdpi.com/1420-3049/19/6/8238/s1.

\section{Acknowledgments}

The publication was supported in part by the Research Grant for Young Investigators of Faculty of Agriculture, Kyushu University.

\section{Author Contributions}

A.T., Q.Z., K.O., H.K. and K.S. designed research; A.T., Q.Z., H.T., H.H., Y.M., R.Y., H.I., A.I. and K.S. performed research and analyzed the data; A.T., Q.Z. and K.S. wrote the paper. All authors read and approved the final manuscript.

\section{Conflicts of Interest}

The authors declare no conflict of interest.

\section{References}

1. Lee, H.-O.; Baek, S.-H.; Han, D.-M. Antimicrobial effects of Chamaecyparis obtusa essential oil. Korean J. Appl. Microbiol. Biotechnol. 2001, 29, 253-257.

2. Zhang, Y.; Jiao, J.; Liu, C.; Wu, X.; Zhang, Y. Isolation and purification of four flavone $\mathrm{C}$-glycosides from antioxidant of bamboo leaves by macroporous resin column chromatography and preparative high-performance liquid chromatography. Food Chem. 2008, 107, 1326-1336.

3. Lu, B.-Y.; Zhang, Y.; Wu, X.-Q. Advances in studies on antioxidative activity and cardiocerebrovascular pharmacology of bamboo-leaf-flavonoids. Linchan Huaxue Yu Gongye 2005, 25, $120-124$.

4. Jiao, J.; Zhang, Y.; Liu, C.; Liu, J.; Wu, X. Separation and purification of tricin from an antioxidant product derived from bamboo leaves. J. Agric. Food Chem. 2007, 55, 10086-10092.

5. Hasegawa, T.; Tanaka, A.; Hosoda, A.; Takano, F.; Ohta, T. Antioxidant C-glycosyl flavones from the leaves of Sasa kurilensis var. gigantea. Phytochemistry 2008, 69, 1419-1424.

6. Zhang, Y.; Yao, X.; Bao, B. Anti-fatigue activity of a triterpenoid-rich extract from Chinese bamboo shavings (Caulis bamfusae in taeniam). Phytother. Res. 2006, 20, 872-876.

7. Kurokawa, T.; Itagaki, S.; Yamaji, T.; Nakata, C.; Noda, T.; Hirano, T.; Iseki, K. Antioxidant activity of a novel extract from bamboo grass (AHSS) against ischemia-reperfusion injury in rat small intestine. Biol. Pharm. Bull. 2006, 29, 2301-2303. 
8. Nishina, A.; Hasegawa, K.I.; Uchibori, T.; Seino, H.; Osawa, T. 2,6-dimethoxy-p-benzoquinone as an antibacterial substance in the culm of Phyllostachys heterocycla var. Pubescens, a species of thick-stemmed bamboo. J. Agric. Food Chem. 1991, 39, 266-269.

9. Fujimura, M.; Ideguchi, M.; Minami, Y.; Watanabe, K.; Tadera, K. Amino acid sequence and antimicrobial activity of chitin-binding peptides, Pp-AMP 1 and Pp-AMP 2, from Japanese bamboo shoots (Phyllostachys pubescens). Biosci. Biotechnol. Biochem.2005, 69, 642-645.

10. Tanaka, A.; Shimizu, K.; Kondo, R. Antibacterial compounds from shoot skins of moso bamboo (Phyllostachys pubescens). J. Wood Sci. 2013, 59, 155-159.

11. Katsuzaki, H.; Sakai, K.; Achiwa, Y.; Imai, K.; Komiya, T. Isolation of antioxidative compounds from bamboo shoots sheath. Nippon Shokuhin Kagaku Kogaku Kaishi 1999, 46, 491-493.

12. Fujita, S. Ecological and mechanical aspect of bamboo. Biol. Resour. 2012, 6, 2-11. (In Japanese).

13. Guo, X.F.; Yue, Y.D.; Tang, F.; Wang, J.; Yao, X. Antioxidant properties of major flavonoids and subfractions of the extract of Phyllostachys pubescens leaves. J. Food Biochem. 2012, 37, 501-509.

14. Zhang, Z.; Wang, X.; Yu, S.; Zhao, M. Isolation and antioxidant activities of polysaccharides extracted from the shoots of Phyllostachys edulis (Carr.). Int J. Biol Macromol 2011, 49, 454-457.

15. Higa, J.K.; Panee, J. Bamboo extract reduces interleukin 6 (IL-6) overproduction under lipotoxic conditions through inhibiting the activation of NF-kappaB and AP-1 pathways. Cytokine 2011, $55,18-23$.

16. Lin, Y.; Collier, A.C.; Liu, W.; Berry, M.J.; Panee, J. The inhibitory effect of bamboo extract on the development of 7,12-dimethylbenz[a]anthracene (DMBA)-induced breast cancer. Phytother Res. 2008, 22, 1440-1445.

17. Tanaka, A.; Kim, H.J.; Oda, S.; Shimizu, K.; Kondo, R. Antibacterial activity of moso bamboo shoot skin (Phyllostachys pubescens) against Staphylococcus aureus. J. Wood Sci. 2011, 57, 542-544.

18. Ferreres, F.; Gil-Izquierdo, A.; Andrade, P.B.; Valentao, P.; Tomas-Barberan, F.A. Characterization of C-glycosyl flavones O-glycosylated by liquid chromatography-tandem mass spectrometry. J. Chromatogr. A 2007, 1161, 214-223.

19. Meng, T.X.; Furuta, S.; Fukamizu, S.; Yamamoto, R.; Ishikawa, H.; Arung, E.T.; Shimizu, K.; Kondo, R. Evaluation of biological activities of extracts from the fruiting body of Pleurotus citrinopileatus for skin cosmetics. J. Wood Sci.2011, 57, 452-458.

20. Breuer, K.; HÄussler, S.; Kapp, A.; Werfel, T. Staphylococcus aureus: Colonizing features and influence of an antibacterial treatment in adults with atopic dermatitis. Br. J. Dermatol. 2002, 147, $55-61$.

21. Iwamoto, A.; Mitsuda, K.; Inoue, A.; Kato, T.; Inoue, Y.; Kawahara, H. Purification and identification of an IgE suppressor from strawberry in an in vitro immunization system. Cytotechnology 2012, 64, 309-314.

22. Van Hoyweghen, L.; de Beer, T.; Deforce, D.; Heyerick, A. Phenolic compounds and anti-oxidant capacity of twelve morphologically heterogeneous bamboo species. Phytochem. Anal. 2012, 23, 433-443.

23. Lv, Z.; Dong, J.; Zhang, B. Rapid identification and detection of flavonoids compounds from bamboo leaves by LC-(ESI)-IT-TOF/MS. BioResources 2012, 7, 1405-1418.

24. Cavaliere, C.; Foglia, P.; Pastorini, E.; Samperi, R.; Lagana, A. Identification and mass spectrometric characterization of glycosylated flavonoids in Triticum durum plants by 
high-performance liquid chromatography with tandem mass spectrometry. Rapid Commun. Mass Spectrom. 2005, 19, 3143-3158.

25. Ferreres, F.; Silva, B.M.; Andrade, P.B.; Seabra, R.M.; Ferreira, M.A. Approach to the study of C-glycosyl flavones by ion trap HPLC-PAD-ESI/MS/MS: Application to seeds of quince (Cydonia oblonga). Phytochem. Anal. 2003, 14, 352-359.

26. Huang, C.S.; Lii, C.K.; Lin, A.H.; Yeh, Y.W.; Yao, H.T.; Li, C.C.; Wang, T.S.; Chen, H.W. Protection by chrysin, apigenin, and luteolin against oxidative stress is mediated by the Nrf2dependent up-regulation of heme oxygenase 1 and glutamate cysteine ligase in rat primary hepatocytes. Arch. Toxicol 2013, 87, 167-178.

27. Birt, D.F.; Walker, B.; Tibbels, M.G.; Bresnick, E. Anti-mutagenesis and anti-promotion by apigenin, robinetin and indole-3-carbinol. Carcinogenesis 1986, 7, 959-963.

28. Funakoshi-Tago, M.; Nakamura, K.; Tago, K.; Mashino, T.; Kasahara, T. Anti-inflammatory activity of structurally related flavonoids, Apigenin, Luteolin and Fisetin. Int. Immunopharmacol 2011, 11, 1150-1159.

29. Czyz, J.; Madeja, Z.; Irmer, U.; Korohoda, W.; Hulser, D.F. Flavonoid apigenin inhibits motility and invasiveness of carcinoma cells in vitro. Int J. Cancer 2005, 114, 12-18.

30. Choi, J.S.; Islam, M.N.; Ali, M.Y.; Kim, E.J.; Kim, Y.M.; Jung, H.A. Effects of C-glycosylation on anti-diabetic, anti-Alzheimer's disease and anti-inflammatory potential of apigenin. Food Chem. Toxicol 2014, 64, 27-33.

31. Iwaoka, E.; Oku, H.; Iinuma, M.; Ishiguro, K. Allergy-preventive effects of the flowers of Impatiens textori. Biol. Pharm. Bull. 2010, 33, 714-716.

32. Kritas, S.K.; Saggini, A.; Varvara, G.; Murmura, G.; Caraffa, A.; Antinolfi, P.; Toniato, E.; Pantalone, A.; Neri, G.; Frydas, S.; et al. Luteolin inhibits mast cell-mediated allergic inflammation. J. Biol. Regul. Homeost. Agents 2013, 27, 955-959.

33. Li, S.Y.; Chang, C.Q. Biological effects of chlorogenic acid and body health. Wei Sheng Yan Jiu 2005, 34, 762-764.

34. Oku, H.; Ogawa, Y.; Iwaoka, E.; Ishiguro, K. Allergy-preventive effects of chlorogenic acid and iridoid derivatives from flower buds of Lonicera japonica. Biol. Pharm. Bull. 2011, 34, 1330-1333.

35. Stermitz, F.R.; Beeson, T.D.; Mueller, P.J.; Hsiang, J.; Lewis, K. Staphylococcus aureus MDR efflux pump inhibitors from a Berberis and a Mahonia (sensu strictu) species. Biochem. Syst. Ecol. 2001, 29, 793-798.

36. Shukla, S.; Gupta, S. Apigenin: A promising molecule for cancer prevention. Pharm. Res. 2010 , 27, 962-978.

37. Romanova, D.; Vachalkova, A.; Cipak, L.; Ovesna, Z.; Rauko, P. Study of antioxidant effect of apigenin, luteolin and quercetin by DNA protective method. Neoplasma 2001, 48, 104-107.

38. Arung, E.T.; Shimizu, K.; Kondo, R. Inhibitory effect of artocarpanone from Artocarpus heterophyllus on melanin biosynthesis. Biol. Pharm. Bull. 2006, 29, 1966-1969.

39. Prior, R.L.; Hoang, H.; Gu, L.; Wu, X.; Bacchiocca, M.; Howard, L.; Hampsch-Woodill, M.; Huang, D.; Ou, B.; Jacob, R. Assays for hydrophilic and lipophilic antioxidant capacity (oxygen radical absorbance capacity (ORAC(FL))) of plasma and other biological and food samples. J. Agric. Food Chem. 2003, 51, 3273-3279. 
40. Nakamura, K.; Ogasawara, Y.; Endou, K.; Fujimori, S.; Koyama, M.; Akano, H. Phenolic compounds responsible for the superoxide dismutase-like activity in high-Brix apple vinegar. J. Agric. Food Chem. 2010, 58, 10124-10132.

41. Re, R.; Pellegrini, N.; Proteggente, A.; Pannala, A.; Yang, M.; Rice-Evans, C. Antioxidant activity applying an improved ABTS radical cation decolorization assay. Free Radic Biol. Med. 1999, 26, 1231-1237.

Sample Availability: Samples of the compounds are available from the authors.

(C) 2014 by the authors; licensee MDPI, Basel, Switzerland. This article is an open access article distributed under the terms and conditions of the Creative Commons Attribution license (http://creativecommons.org/licenses/by/3.0/). 